\title{
Measurements of the optical coefficients of the protoporphyrin IX endogenously producing yeast-based model in the visible and NIR
}

\author{
Jaroslava Joniová \\ Veronika Kažiková \\ Emmanuel Gerelli \\ Gregor Bánó \\ Georges Wagnières
}




\title{
Measurements of the optical coefficients of the protoporphyrin IX endogenously producing yeast-based model in the visible and NIR
}

\author{
Jaroslava Joniová,, ${ }^{\mathrm{a} *}$ Veronika Kažiková, ${ }^{\mathrm{b}}$ Emmanuel Gerelli, ${ }^{a}$ Gregor Bánó, ${ }^{\mathrm{b}, \mathrm{c}}$ and Georges Wagnières ${ }^{\mathrm{a}}$ \\ ${ }^{a}$ Swiss Federal Institute of Technology (EPFL), Institute of Physics, Laboratory for Functional and Metabolic Imaging, Lausanne, Switzerland \\ ${ }^{\mathrm{b}}$ P.J. Šafárik University, Institute of Physics, Department of Biophysics, Faculty of Science, Košice, Slovakia \\ 'P.J. Šafárik University, Center for Interdisciplinary Biosciences, Technology and Innovation Park, Košice, Slovakia
}

\begin{abstract}
Models mimicking the endogenous production of protoporphyrin IX ( $\mathrm{PpIX})$, as well as its fluorescence, are of high interest for applied and fundamental studies in the fields of cancer detection by fluorescence imaging, photodynamic therapy (PDT), and photobiomodulation (PBM). Here, we present and describe optical properties of the yeast-based models able to produce PpIX endogenously after the administration of 5-aminolevulinic acid (ALA) and/or 2,2'-bipyridyl. As their optical properties have an important impact on the spatial distribution of the fluence rate in these liquid models, their absorption and reduced scattering coefficients were determined to be between 400 and $808 \mathrm{~nm}$ for two yeast solutions previously described by our group. These coefficients were derived from measurements of the total reflectance and light penetration depth using a dedicated Monte Carlo simulation. We observed that absorption and scattering coefficients were smaller than those of soft tissues at all wavelengths. This work will enable the production of a low-cost optical phantom loaded with appropriate amounts of light-absorbing and -scattering particles to mimic tumors containing PpIX, offering a useful tool to optimize the spectral and radiometric design of certain cancer photodetection setups. $\odot 2018$ Society of PhotoOptical Instrumentation Engineers (SPIE) [DOI: 10.1117/1.JBO.23.7.075001]
\end{abstract}

Keywords: optical phantom; yeast; optical coefficients; protoporphyrin IX; endogenous production; 5-aminolevulinic acid. Paper 180132R received Mar. 5, 2018; accepted for publication Jun. 13, 2018; published online Jul. 6, 2018.

\section{Introduction}

A precise control of light dosimetry is frequently of utmost importance in biomedical optics and photomedicine. This is, in particular, the case in photodynamic therapy $(\mathrm{PDT})^{1}$ and photobiomodulation $(\mathrm{PBM}){ }^{2}$ as well as for certain diagnostic applications of light, including cancer photodynamic detection (PDD). ${ }^{3}$ As a direct measurement of the fluence rate is often difficult to conduct, different light propagation models requiring knowledge of the tissue optical properties have been developed. ${ }^{1,4}$ These properties can be described by the wavelength-dependent absorption and reduced scattering coefficients, as well as the refractive index $(n) .{ }^{4}$ The optimal approaches to measure these coefficients depend, among others, on the sample geometry and access. One convenient and reliable method consists of measuring the total reflectance $\left(R_{d}\right)$ and light penetration depth (LPD) in the sample, two parameters from which the optical coefficients mentioned above can be derived by solving the inverse problem. ${ }^{5}$ This approach is well suited for liquid optical phantoms, as is the case for the yeast-based models we described previously. ${ }^{6,7}$ This convenient, reproducible, and inexpensive model organism can generate endogenously predictable levels of protoporphyrin IX (PpIX), a feature that is of interest in various fields of photomedicine, such as PDT, PDD, and PBM. Indeed, PpIX produced in the mitochondria is one of the most extensively used photosensitizers (PS) for

*Address all correspondence to: Jaroslava Joniová, E-mail: jaroslava.joniova@ epfl.ch
PDT. Most cells endogenously produce this photoactive, oxygen-sensitive, and fluorescing PS through the biosynthetic pathway of heme. ${ }^{8}$ In most cells, PpIX production can be significantly enhanced following the administration of its precursor, 5-aminolevulinic acid (ALA), or some of its derivatives. ${ }^{9-11}$ ALA-based treatments and diagnostic procedures (PDT, PDD, and fluorescence-guided surgery) are widely used in dermatology, neurosurgery, and urology. ${ }^{3,12-14}$ In addition, as PBM is known to modulate the metabolism, measuring its effects on the endogenous production of PpIX provides information of fundamental interest. ${ }^{15}$ Thus, the development of such optical phantoms with known optical properties and mimicking the endogenous production of PpIX, as well as its fluorescence, is of great interest for fundamental and experimental studies in relation with photobiological studies including PpIX-based PDT, PDD, and PBM.

Therefore, the main aim of this study was to determine the optical properties of yeast-based model systems described in our previous reports at wavelengths of interest for PDT, PBM, and PDD, i.e., between 400 and $808 \mathrm{~nm}$. To the best of our knowledge, this is the first time that the optical properties are reported for an optical phantom and model organism able to produce PpIX endogenously. We measured both $R_{d}$ and the LPD when the yeast solutions were illuminated with a "broad" (diameter: $15 \mathrm{~cm}$ ) light beam, and the absorption $\left(\mu_{a}\right)$ and reduced scattering $\left(\mu_{s}^{\prime}\right)$ coefficients were then deduced with a dedicated Monte Carlo algorithm. 
We have conducted these measurements for two types of aqueous yeast solutions, i.e., in yeast extract-peptone-dextrose (YPD) or in a glucose solution. In addition to their different optical properties, these environments induce different yeast metabolic activities and replication rates. ${ }^{6,7}$

\section{Material and Methods}

\subsection{Chemicals}

All chemicals were purchased from Sigma Aldrich, Switzerland.

\subsection{Yeast Growing Conditions}

Two different growth conditions for yeast cultivation were used in this study. About $2.5 \mathrm{mg} / \mathrm{mL}$ Saccharomyces cerevisiae YSC2 (type II) was dissolved either in a standardly used YPD solution $(50 \mathrm{mg} / \mathrm{mL})$ or in an aqueous glucose $(150 \mathrm{mg} / \mathrm{mL})$ solution. ${ }^{6,7}$ Yeast samples were incubated in a thermostabilized $\left(32^{\circ} \mathrm{C}\right)$ rotary shaker $(210 \mathrm{rpm})$ for $3 \mathrm{~h}$ until further use.

\subsection{Experimental Measurements of the Optical Properties $\left(R_{d}, L P D\right.$, and $\left.n\right)$}

The setup shown in Fig. 1 was used to measure the fluence rate and $R_{d}$ in yeast solutions. An argon ion $(457,488$, and $514 \mathrm{~nm})$ and a frequency-doubled YAG $(532 \mathrm{~nm})$ lasers (SpectraPhysics, Mountain View, California; Model 2020 and Millennia, respectively) were used as light sources for the measurements. Diode lasers were used for all other wavelengths (typical bandwidth $<5 \mathrm{~nm}$ ): $405 \mathrm{~nm}$ was generated by a source from Oxxius SA, France, $635 \mathrm{~nm}$ was produced by a diode laser from CeramOptec, Germany, whereas 730 and $808 \mathrm{~nm}$ were produced by diode lasers provided by Roithner LaserTechnik $\mathrm{GmbH}$, Austria. The light delivered by these light sources was coupled in the SMA connector of a frontal light distributor

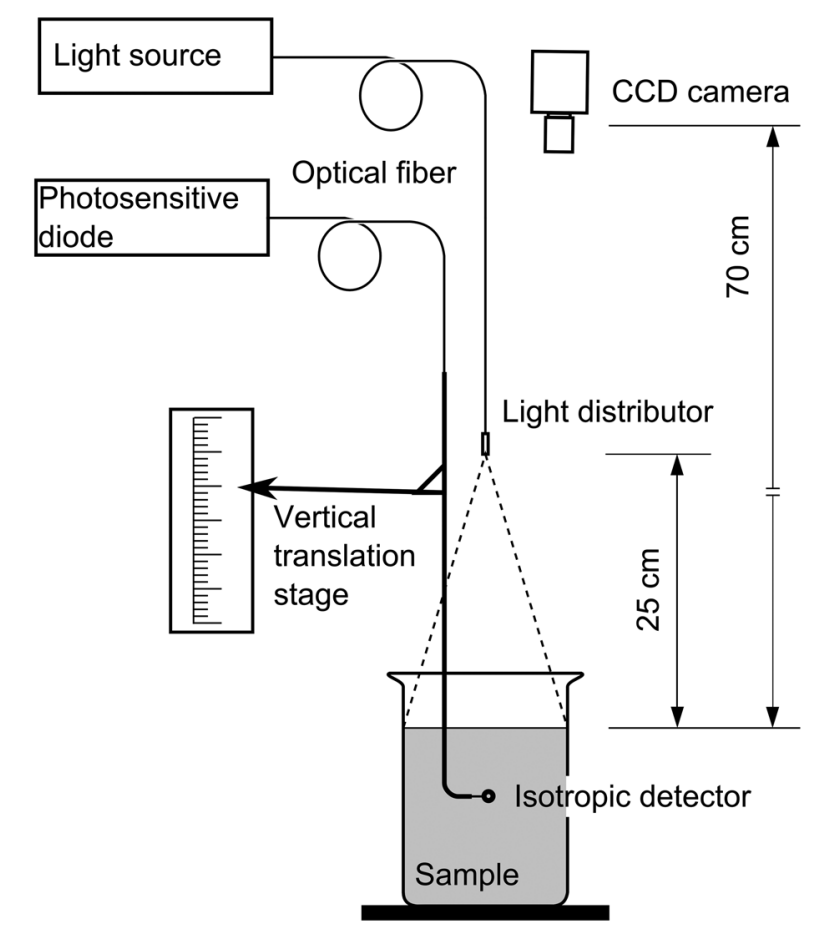

Fig. 1 Sketch of the experimental setup used to measure the optical properties of the yeast solution models.
(Model FD 1, Medlight SA, Switzerland; 2-mm outer diameter) producing a circular (diameter: $15 \mathrm{~cm}$ ) homogenous spot with sharp edges. The powers delivered by these light distributors ranged between 50 and $500 \mathrm{~mW}$, depending on the detector sensitivity and dynamic ranges.

Yeast solutions (in glucose or YPD) were prepared according to the protocol described above and placed in a 3-L cylindrical borosilicate vessel with the following geometry: (height: $200 \mathrm{~mm}$; diameter: $150 \mathrm{~mm}$; wall thickness: $3.5 \mathrm{~mm}$ ). A black velvet mat was put underneath the vessel to absorb the photons reaching the bottom of the vessel. The fluence rate was measured using a scattering spherical isotropic probe (Model IP, Medlight SA, Switzerland; light detection sphere diameter: $850 \mu \mathrm{m})$ attached to a translation stage enabling a vertical displacement. This isotropic probe was inserted into a stainless steel tube (outer diameter: $1 \mathrm{~mm}$ ) to prevent a direct coupling of the light in the optical fiber collecting the light from the scattering sphere. The lower part of this tube was bended at $90 \mathrm{deg}$ to orient optimally the scattering sphere and to prevent possible artifacts induced by the shadow produced by this fiber holder. This tube was covered with a Kodak white reflectance coating (Eastman Kodak Company), a nonfluorescent coating backscattering $>98 \%$ of the light between 300 and $1200 \mathrm{~nm}$, to prevent changes of the photon distribution in the solution due to the limited reflectivity of stainless steel. For the measurements at 405 nm, a MV405/20 - Narrow Violet Vision Filter (Chroma Technology Corp.) was positioned between the SMA connector of the fiber guiding the light from the isotropic probe and a photosensitive diode (DET 210, ThorLabs) to reject the fluorescence induced by violet light in the yeast solutions.

The fluence rate was measured six times every $5 \mathrm{~mm}$ from the top to the bottom of the vessel, and vice versa. The effective attenuation coefficient $\left(\mu_{\text {eff }}\right)$ can, in principle, be derived from such measurements by a mono-exponential fit corresponding to the decrease in the fluence rate with depth, ${ }^{5,16-18}$ if the illumination is broad enough and if the hypothesis that is at the basis of the diffusion approximation is satisfied (diffuse light flux; $\left.\mu_{a} \ll \mu_{s}^{\prime}\right)$. Although most of these conditions were satisfied in our measurements, edge effects were present in most situations as, in the worst situation, the illumination spot was only about three times larger than $\mu_{\text {eff }}^{-1}$. In the other words, the illumination spot could not be considered as semi-infinite. This is the reason why the parameters extracted from our depth measurements of the fluence rate is called "light penetration depth" (LPD) in the following text. The LPD was defined as the inverse of the slope of the linear fit representing the logarithmic decay of the fluence rate measured as a function of depth (Fig. 2). Only the points at which the regression line was within the error bars were considered to determine the slope. This approach was used to reject the points measured close to the surface, where the diffusion approximation does not apply.

The same experimental setup (Fig. 1) was used to assess the total reflectance $R_{d}$. A scientific CCD camera (Hamamatsu, EM-CCD C9100 equipped with a Fujinon TV ZOOM LENS 1:1.2/12.5-75 mm H6x12.5R - MD 3) was positioned $70 \mathrm{~cm}$ above the surface of the yeast solution with an angle of $8 \mathrm{deg}$ relative to the perpendicular to the air-solution surface to minimize the specular reflections. For each picture and wavelength, a region of interest (ROI) was selected while avoiding specular reflections, and the brightness was determined with the software ImageJ (National Institutes of Health, Bethesda, 


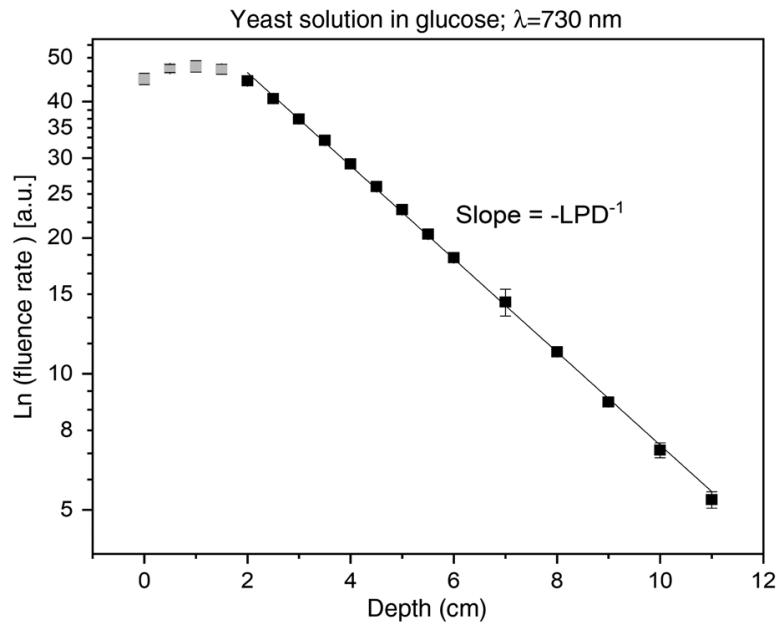

Fig. 2 Illustrative measurement of the fluence rate at different depths. Points (in gray) close to the surface were not considered to determine the slope, i.e., only points (in black) at which regression line was within the error bars are considered to determine the slope. Error bars represent the standard deviation of six individual measurements.

Maryland). The signal acquired in the dark (background) was measured using the same ROI and subtracted from all measurements. At each wavelength, the absolute value of $R_{d}$ was determined by comparing the brightness obtained with the yeast solutions and when a diffuse reflectance standard (SphereOptics GmbH, Germany), reflecting $>99 \%$, was placed at the position corresponding to the yeast solution surface. The refractive index of yeast in glucose or YPD solutions was determined at different wavelengths ranging between 405 and $808 \mathrm{~nm}$ at room temperature according to the following procedure. An Abbe's refractometer (OPL, France) was used to first determine the refractive index of distilled water at $589 \mathrm{~nm}$. Our obtained results were in agreement with those reported in the literature. ${ }^{19}$ We then measured the refractive index of the yeast solutions that differed from that of distilled water at this wavelength. Because our refractometer is designed to measure the refractive index at $589 \mathrm{~nm}$ only, we estimated the values of this parameter at other wavelengths by adding this difference to the values reported in the literature for water.

\subsection{Monte Carlo Simulation to Determine $\mu_{a}$ and $\mu_{s}^{\prime}$}

As mentioned above, if $\mu_{a}$ is much smaller than $\mu_{s}^{\prime}$, these microscopic optical parameters can be calculated from $R_{d}$ and the LPD using the analytical equation derived in the framework of light diffusion approximation. ${ }^{16,20}$ However, as shown below in the results and discussion section, the assumption $\mu_{a} \ll \mu_{s}^{\prime}$ required for the diffusion theory to be valid is not satisfied for the present yeast solutions at all wavelengths. In addition, using the experimental arrangement described above, the effect of the container walls on the light penetration cannot be neglected. Therefore, the optical parameters of the yeast solutions were determined using a Monte Carlo-based ray tracing software (TracePro; Lambda Research Corporation).

The geometry of the modeled system, including the container and the illumination parts, was matched with the experimental setup. On the basis of preliminary studies, the number of photons followed in each simulation was set to $1.2 \times 10^{7}$. In addition, the Henyey-Greenstein phase function was used to approximate the scattering processes, the corresponding average cosine being set to $g=0.95$. The experimental refractive index values were implemented in the model.

Fifteen virtual spherical probes (diameter $2 \mathrm{~mm}$ ) separated by $10 \mathrm{~mm}$ were positioned along the optical axis inside the yeast solution to monitor the light fluence-rate at different depths. The LPD was calculated from the exponentially decaying part of the fluence-rate spatial distribution, typically in the depth range of 90 to $150 \mathrm{~mm}$ (Fig. 2). The reflectance $R_{d}$ was obtained by counting the photons passing through a circular probe surface (diameter $20 \mathrm{~mm}$ ) placed $0.1 \mathrm{~mm}$ above the solution level at the container center (ROI). To mimic the light collected by the CCD camera in the experimental setup, only the photons propagating in the upward direction at $8 \mathrm{deg} \pm 0.5 \mathrm{deg}$, with respect to the optical axis, were taken into account. The reference number of reflected photons was obtained when the solutions were replaced by a totally reflecting Lambertian surface predefined in the TracePro software.

For each experimental condition, the optical parameters of the yeast model were determined by an iterative process. After choosing an initial guess for $\mu_{a}$ and $\mu_{s}^{\prime}$, the GaussNewton method ${ }^{21}$ was applied consecutively to minimize the sum of squared relative differences (RD)

$S=\mathrm{RD}_{1}^{2}+\mathrm{RD}_{2}^{2}=\left(\frac{R_{d}^{\mathrm{sim}}-R_{d}^{\exp }}{R_{d}^{\exp }}\right)^{2}+\left(\frac{\mu_{\mathrm{eff}}^{\mathrm{sim}}-\mu_{\mathrm{eff}}^{\exp }}{\mu_{\mathrm{eff}}^{\exp }}\right)^{2}$.

The use of relative differences compensates for the disproportion of the reflectance and LPD numerical values and ensures the same upper limit for their relative errors. In each iteration step of the Gauss-Newton method, the Jacobian matrix of the RD vector [Eq. (1)] with respect to $\mu_{a}$ and $\mu_{s}^{\prime}$ was determined. This matrix required Monte Carlo simulations at three different $\left(\mu_{a}, \mu_{s}^{\prime}\right)$ pairs. The time needed for a single Monte Carlo simulation was $\sim 45 \mathrm{~min}$. The iterations were stopped when $S<0.0002$ was reached. It follows that the highest possible difference between the experimental and the converged simulated values of $R_{d}$ or LPD was $1.4 \%$ (the square root of 0.0002). Typically, 3 to 6 iteration steps (each consisting of three simulations) were needed to reproduce one experimental condition (wavelength).

\section{Results and Discussion}

The optical parameters of yeast-based model systems able to produce endogenously PpIX ${ }^{6,7}$ were determined in this study. The precise determination of these properties plays one of the most important steps to predict the light dosimetry. As we will see below, the yeast solutions are rather transparent with optical coefficients quite different from those of soft tissues. However, this is not an issue for many applications of these model systems, as the concept of interest is the knowledge and control the fluence rate everywhere in the solution, i.e., to expose the yeast with known light doses. It should be noted that, if necessary, the addition of absorbing and scattering particles can change the optical properties of such yeast solutions in such a way that they would correspond to those of soft tissues. This is of interest in the fields of PpIX-based PDT, PDD, or PBM, if such solutions are used, for example, as optical phantom mimicking tumors producing PpIX. These particles must be inert in the sense that they must not affect the yeast metabolism. 
LPD and $R_{d}$ measurements were conducted for yeast grown in two types of aqueous environments: in glucose or YPD solution. In addition to their different optical properties, these environments induce different yeast metabolic activities and replication rates, i.e., in the presence of standardly used YPD solution, yeast cells are dividing causing a change of oxygen partial pressure $\left(\mathrm{pO}_{2}\right)$ in the medium $\left(\mathrm{pO}_{2}\right.$ decreases with an increase in the yeast concentration due to cell respiration). Moreover, due to the replication of yeast, there is a higher yeast concentration in the YPD solution causing different scatterings of the sample. In contrary, yeast grown in an aqueous glucose does not replicate, which leads to a more constant $\mathrm{pO}_{2}$. It is worth mentioning that the absence of replication of yeast cells in an aqueous glucose does not preclude the endogenous PpIX production after administration of ALA and/or 2,2'-bipyridyl. ${ }^{6}$ Moreover, for both environments, the fluorescence brightness of PpIX produced endogenously in yeast was comparable (i.e., of the same order of magnitude) to the PpIX fluorescence brightness observed in actinic keratosis. 7,11

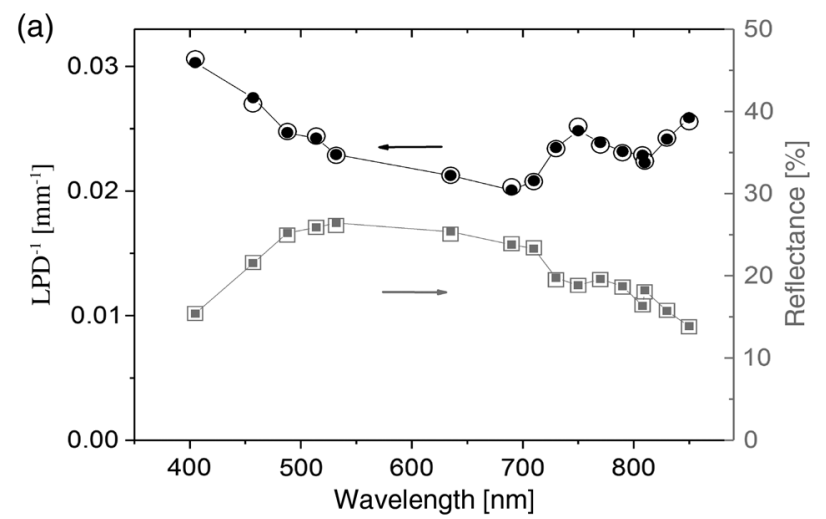

\subsection{Experimental Estimation of the $L P D, R_{d}$, and the Refractive Index in Yeast Solutions}

The values of $\mathrm{LPD}^{-1}$ and $R_{d}$ assessed experimentally using the setup shown in Fig. 1, which are shown in Fig. 3 for both (glucose and YPD) solutions. The wavelength dependence of these parameters varies significantly between the two solutions, yeast in YPD presenting the shorter penetration depth at short wavelengths. This spectral evolution is probably due to the Soret band of heme derivatives that are more abundant in YPD. The large LPDs (up to $50 \mathrm{~mm}$ ) measured in the red and near infrared illustrate that our vessel, although relatively large (diameter: $150 \mathrm{~mm}$; depth: $150 \mathrm{~mm}$ ), cannot be considered as "semi-infinite," a situation that explains why the optical coefficients have been derived with a Monte Carlo-based simulation. The values of $\mathrm{LPD}^{-1}$ and $R_{d}$ resulting from the optical coefficients derived by the simulation are also shown in Fig. 3.

The excellent match between the measured and calculated values of $\mathrm{LPD}^{-1}$ and $R_{d}$ shows the accuracy of the computation method mentioned above.

Fig. 3 Experimental (full signs) and simulated values (open signs) of $\mathrm{LPD}^{-1}$ and $R_{d}$ for yeast in (a) glucose and (b) YPD.

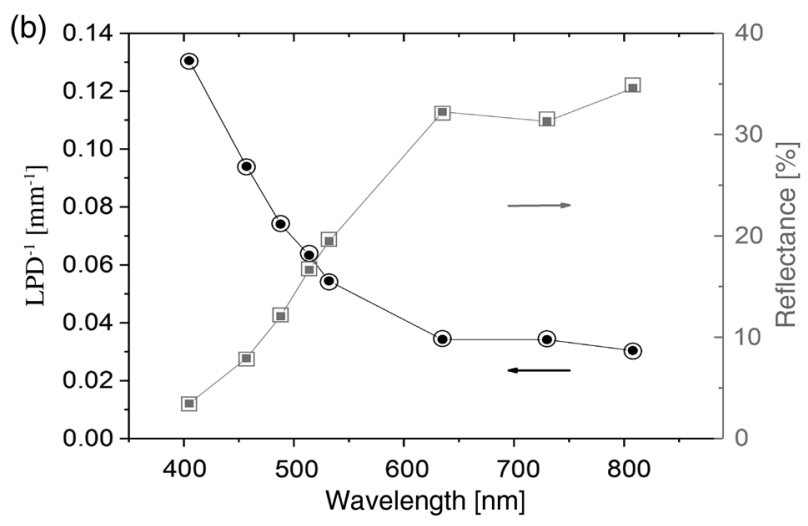

(a)

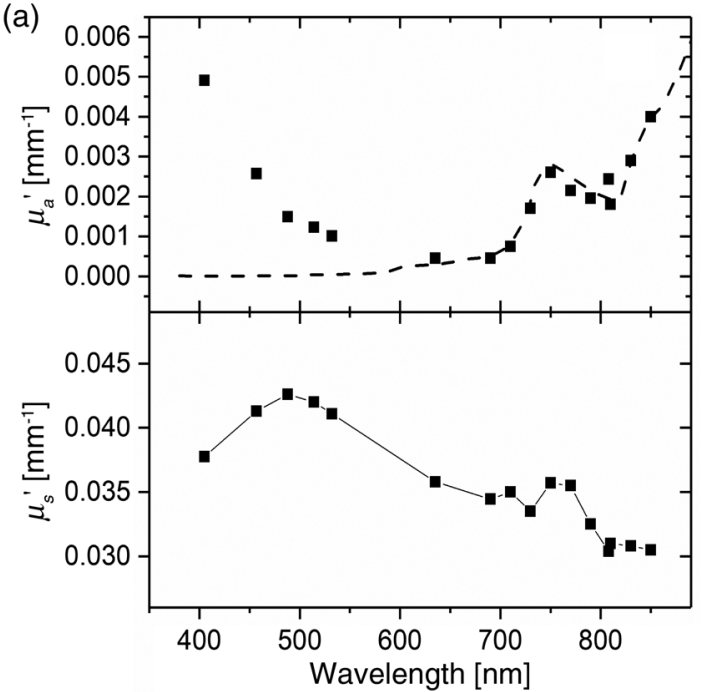

(b)

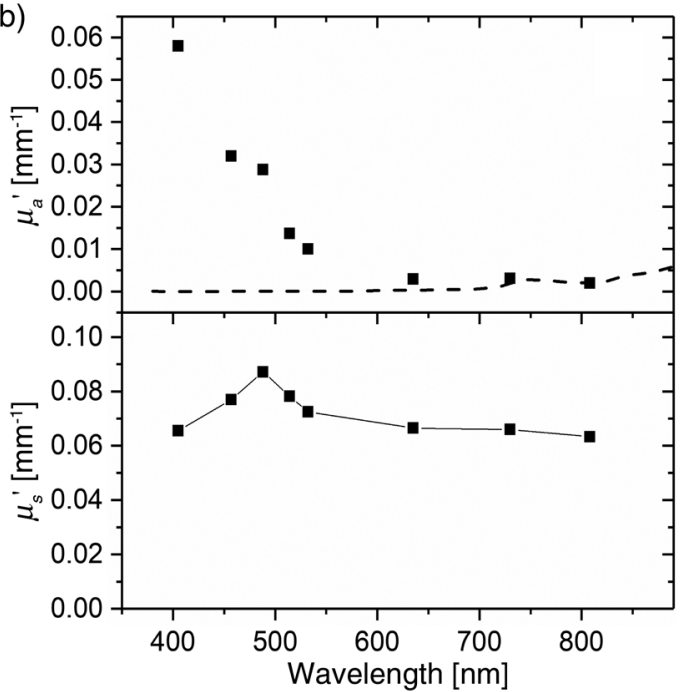

Fig. 4 Estimated optical parameters $\mu_{a}$ and $\mu_{s}^{\prime}$ yeast in (a) glucose and (b) YPD (both black squares); $\mu_{a}$ of distilled water is indicated by the dashed line, whereas the lines presented for $\mu_{s}^{\prime}$ is for visual support only. 
The refractive indices we have measured at $589 \mathrm{~nm}$ and at room temperature were 1.337 and 1.352 for the YPD and glucose solutions, respectively. These values are close to that of pure water and of a $150 \mathrm{mg} / \mathrm{mL}$ glucose solution, respectively. This is not surprising as yeast cells are quite diluted $(2.5 \mathrm{mg} / \mathrm{mL})$ and consist mostly of water $(70 \%) .^{22}$

\subsection{Computational Estimation of $\mu_{a}$ and $\mu_{s}^{\prime}$}

The refractive index of both yeast solutions was assessed for other wavelengths, but $589 \mathrm{~nm}$ was used as mentioned above to perform the simulations. Our estimated refractive index at other wavelengths in glucose solution led to results that are consistent with the literature. ${ }^{23}$

The values we obtained for $\mu_{a}$ and $\mu_{s}^{\prime}$ between 400 and $850 \mathrm{~nm}$ are shown in Figs. 4(a) and 4(b) for yeast in glucose and YPD solutions, respectively.

It is interesting to note that the absorption at wavelengths $>600 \mathrm{~nm}$ is mostly due to water. The excellent match between our measurements and the absorption of water in this spectral domain, which contains an overtone of a vibrational band around $750 \mathrm{~nm}$, validates our experimental and data analysis procedure. $^{24,25}$ Thus, it can be concluded that in this spectral region, the yeast absorption is negligible. Finally, the wavelength dependence of $\mu_{s}^{\prime}$ is relatively weak, a result that can be expected considering that yeast cells are diluted and relatively large as compared with the wavelength. ${ }^{26}$

\section{Conclusion}

The main objective of this study was to assess the optical properties of yeast-based model systems able to produce PpIX endogenously. The LPD and $R_{d}$ were assessed experimentally, whereas $\mu_{a}$ and $\mu_{s}^{\prime}$ were deduced by Monte Carlo simulations. We have demonstrated that the results obtained by such simulations are in excellent agreement with those obtained experimentally (for LPD and $R_{d}$ ). Interestingly, the quantitative assessment of the microscopic optical coefficients with our approach is validated by the excellent match existing between the wavelength dependence of $\mu_{a}$ and the absorption coefficient of water above $600 \mathrm{~nm}$. One interesting feature of our system is that, when loaded with appropriate amounts of absorbing and scattering particles, it can be used as optical phantom mimicking tumors containing PpIX produced endogenously, a very useful tool to study and optimize PpIX-based phototreatment and photodiagnosis.

\section{Disclosures}

GW is a shareholder of Medlight SA, a company providing light distributors and isotropic detectors. Authors have no other conflict of interest to declare.

\section{Acknowledgments}

This work was supported by the Swiss National Science Foundation (Grants No. 205320-116556 and CR32I3_159746) and funded in part by FP7 EU (CELIM 316310).

\section{References}

1. B. C. Wilson and M. S. Patterson, "The physics, biophysics and technology of photodynamic therapy," Phys. Med. Biol. 53(9), R61-R109 (2008).
2. M. R. Hamblin, T. Agrawal, and M. de Sousa, Handbook of Low-Level Laser Therapy, CRC Press, New York (2016).

3. G. Wagnieres et al., "Detection of bladder cancer by fluorescence cystoscopy: from bench to bedside- the HEXVIX story," in Handbook of Photomedicine, M. R. Hamblin and Y. Y. Huang, Eds., pp. 411-425, CRC Press, Boca Raton, Florida (2014).

4. S. L. Jacques, "Optical properties of biological tissues: a review," Phys. Med. Biol. 58(11), R37-R61 (2013).

5. G. Wagnières et al., "An optical phantom with tissue-like properties in the visible for use in PDT and fluorescence spectroscopy," Phys. Med. Biol. 42(7), 1415-1426 (1997).

6. J. Joniová et al., "Optimization and characterization of the endogenous production of protoporphyrin IX in a yeast model," J. Biomed. Opt. 21(12), 125008 (2016).

7. J. Joniová, E. Gerelli, and G. Wagnières, "Modulation of the endogenous production of protoporphyrin IX in a yeast-based model organism," Proc. SPIE 10060, 1006018 (2017).

8. N. Fotinos et al., "5-Aminolevulinic acid derivatives in photomedicine: characteristics, application and perspectives," Photochem. Photobiol. 82(4), 994-1015 (2006).

9. J. T. Lathrop and M. P. Timko, "Regulation by heme of mitochondrial protein transport through a conserved amino acid motif," Science 259(5094), 522-525 (1993).

10. P. Agostinis et al., "Photodynamic therapy of cancer: an update," $C A$ Cancer J. Clin. 61(4), 250-281 (2011).

11. F. Piffaretti et al., "Correlation between protoporphyrin IX fluorescence intensity, photobleaching, pain and clinical outcome of actinic keratosis treated by photodynamic therapy," Dermatology 227(3), 214-225 (2013).

12. D. W. Roberts et al., "Glioblastoma multiforme treatment with clinical trials for surgical resection (aminolevulinic acid)," Neurosurg. Clin. N. Am. 23(3), 371-377 (2012).

13. W. Stummer et al., "Fluorescence-guided surgery with 5-aminolevulinic acid for resection of malignant glioma: a randomised controlled multicentre phase III trial," Lancet Oncol. 7(5), 392-401 (2006).

14. P. A. Valdés et al., "Quantitative fluorescence in intracranial tumor: implications for ALA-induced PpIX as an intraoperative biomarker," J. Neurosurg. 115(1), 11-17 (2011).

15. S. K. Bisland et al., "Increased expression of mitochondrial benzodiazepine receptors following low-level light treatment facilitates enhanced protoporphyrin IX production in glioma-derived cells in vitro," Lasers Surg. Med. 39(8), 678-684 (2007).

16. A. Pitzschke et al., "Red and NIR light dosimetry in the human deep brain," Phys. Med. Biol. 60(7), 2921-2937 (2015).

17. S. A. Prahl, "A Monte Carlo model of light propagation in tissue," Proc. SPIE 10305, 1030509 (1989).

18. S. L. Jacques, "How tissue optics affect dosimetry of photodynamic therapy," J. Biomed. Opt. 15(5), 051608 (2010).

19. D. J. Segelstein, "The complex refractive index of water," Thesis, University of Missouri, Kansas City (1981).

20. S. T. Flock et al., "Monte Carlo modeling of light propagation in highly scattering tissues. I. Model predictions and comparison with diffusion theory," IEEE Trans. Biomed. Eng. 36(12), 1162-1168 (1989).

21. J. Nocedal and S. Wright, Numerical Optimization, 2nd ed., SpringerVerlag, New York (2006)

22. B. Alberts et al., Molecular Biology of the Cell, 4th ed., Garland Science, New York (2002).

23. H. Sobral and M. Peña-Gomar, "Determination of the refractive index of glucose-ethanol-water mixtures using spectroscopic refractometry near the critical angle," Appl. Opt. 54(28), 8453-8458 (2015).

24. R. M. Pope and E. S. Fry, "Absorption spectrum (380-700 nm) of pure water. II. Integrating cavity measurements," Appl. Opt. 36(33), 87108723 (1997).

25. K. F. Palmer and D. Williams, "Optical properties of water in the near infrared," J. Opt. Soc. Am. 64(8), 1107-1110 (1974).

26. M. A. Malone, "Infrared microspectroscopy: a study of the single isolated bread yeast cell," Electronic Thesis or Dissertation, The Ohio State University, https://etd.ohiolink.edu/ (2010).

Biographies for the authors are not available. 